\title{
The impact of fluctuations and correlations in droplet growth by collision-coalescence revisited - Part 1: Numerical calculation of post-gel droplet size distribution
}

\author{
Lester Alfonso $^{1}$ and Graciela B. Raga $^{2}$ \\ ${ }^{1}$ Universidad Autónoma de la Ciudad de México, Mexico City, 09790, México \\ ${ }^{2}$ Centro de Ciencias de la Atmósfera, UNAM, Mexico City, 04510, México \\ Correspondence to: Lester Alfonso (lesterson@yahoo.com)
}

Received: 16 October 2016 - Discussion started: 29 November 2016

Revised: 6 April 2017 - Accepted: 19 April 2017 - Published: 13 June 2017

\begin{abstract}
The impact of stochastic fluctuations in cloud droplet growth is a matter of broad interest, since stochastic effects are one of the possible explanations of how cloud droplets cross the size gap and form the raindrop embryos that trigger warm rain development in cumulus clouds. Most theoretical studies on this topic rely on the use of the kinetic collection equation, or the Gillespie stochastic simulation algorithm. However, the kinetic collection equation is a deterministic equation with no stochastic fluctuations. Moreover, the traditional calculations using the kinetic collection equation are not valid when the system undergoes a transition from a continuous distribution to a distribution plus a runaway raindrop embryo (known as the sol-gel transition). On the other hand, the stochastic simulation algorithm, although intrinsically stochastic, fails to adequately reproduce the large end of the droplet size distribution due to the huge number of realizations required. Therefore, the full stochastic description of cloud droplet growth must be obtained from the solution of the master equation for stochastic coalescence.

In this study the master equation is used to calculate the evolution of the droplet size distribution after the sol-gel transition. These calculations show that after the formation of the raindrop embryo, the expected droplet mass distribution strongly differs from the results obtained with the kinetic collection equation. Furthermore, the low-mass bins and bins from the gel fraction are strongly anticorrelated in the vicinity of the critical time, this being one of the possible explanations for the differences between the kinetic and stochastic approaches after the sol-gel transition. Calcula-
\end{abstract}

tions performed within the stochastic framework provide insight into the inability of explicit microphysics cloud models to explain the droplet spectral broadening observed in small, warm clouds.

\section{Introduction}

Although rain has been observed to form in warm cumulus clouds within about $20 \mathrm{~min}$, calculations that represent condensation and coalescence accurately in such clouds have had difficulty producing rainfall in such a short time except via processes involving giant cloud condensation nuclei (with diameters larger than $2 \mu \mathrm{m}$ ). One of the possible origins of this discrepancy is the stochastic nature of the collision coalescence process that is not well reflected in current models that rely almost exclusively on the kinetic collection (or Smoluchowski) equation, hereafter referred to as KCE (Pruppacher and Klett, 1997):

$$
\begin{aligned}
\frac{\partial N(i, t)}{\partial t} & =\frac{1}{2} \sum_{j=1}^{i-1} K(i-j, j) N(i-j) N(j) \\
& -N(i) \sum_{j=1}^{\infty} K(i, j) N(j),
\end{aligned}
$$

where $N(i, t)$ is the concentration of droplets in bin $i$ and $K(i, j)$ is the collection kernel for droplets in bins $i$ and $j$. Additionally, Eq. (1) fails to represent the droplet size distribution at the time at which raindrop embryos are formed 
(Alfonso et al., 2008), as there is a transition from a continuous distribution to a continuous distribution plus a massive raindrop embryo (i.e, the "runaway droplet"). At that point, the infinite system exhibits a sol-gel transition (also called gelation and in which the runaway droplet is labeled "gel"), the KCE breaks down and the total mass of the system calculated according to the KCE is no longer conserved.

One way to avoid this problem is to adopt the stochastic finite volume description of the coalescence process by using the stochastic simulation algorithm proposed by Gillespie (1975). The stochastic simulation algorithm (hereafter referred as SSA), correctly accounts for fluctuations and correlations, and has been used in cloud simulation studies with realistic collection kernels (Valioulis and List, 1984). However, the SSA has difficulties in accurately reproducing the large end of the droplet size distribution. This is due to the huge number of realizations required to obtain smooth behavior at the large end of the droplet size distribution (Alfonso, 2015). The alternative approach (within the stochastic framework) is to use the master equation:

$$
\begin{aligned}
\frac{\partial P(\bar{n})}{\partial t} & =\sum_{i=1}^{N} \sum_{j=i+1}^{N} K(i, j)\left(n_{i}+1\right)\left(n_{j}+1\right) \\
& \times P\left(\ldots, n_{i}+1, \ldots, n_{j}+1, \ldots, n_{i+j}-1, \ldots ; t\right) \\
& +\sum_{i=1}^{N} \frac{1}{2} K(i, i)\left(n_{i}+2\right)\left(n_{i}+1\right) \\
& \times P\left(\ldots, n_{i}+2, \ldots, n_{2 i}-1, \ldots ; t\right) \\
& -\sum_{i=1}^{N} \sum_{j=i+1}^{N} K(i, j) n_{i} n_{j} P(\bar{n} ; t) \\
& -\sum_{i=1}^{N} \frac{1}{2} K(i, i) n_{i}\left(n_{i}-1\right) P(\bar{n} ; t) .
\end{aligned}
$$

The master Eq. (2) is a gain-loss equation for the probability of each state $P(\bar{n})$. The sum of the first two terms is the gain due to transition from other states, and the sum of the last two terms is the loss due to transitions into other states. This formulation was introduced in the pioneer works of Marcus (1968) and Bayewitz et al. (1974), and was studied in detailed by Lushnikov $(1978,2004)$ and Tanaka and Nakazawa (1993). However, these studies only offer analytical results for a limited number of cases (with constant, sum and product kernels), for monodisperse initial conditions. Furthermore, most of these studies are limited to nongelling conditions and do not provide a coherent framework for the general case.

The exception are the methods developed by Lushnikov (2004) from the analytical solution of the master equation, and more recently by Matsoukas (2015), the later based on arguments from statistical physics. These methods, although also limited to very special cases (product kernel and monodisperse initial conditions), are capable of obtaining solutions in the post-gel regime. For example, in Lush- nikov $(1978,2004)$, the coalescence process takes place in a system with a finite volume that includes a finite number of particles. Within this approach any losses of mass are, by definition, excluded. In the infinite system described by the KCE (Eq. 1), the coagulation process instantly transfers mass to the gel, while in the finite system the gel coalesces with smaller particles, decreasing their concentration - not instantly by rather in a finite time.

In order to study the droplet size distribution after the formation of raindrop embryos (sol-gel transition), for systems with kernels relevant to cloud physics and arbitrary initial conditions, we must rely on numerical methods that are capable of solving the master equation (Eq. 2). We can address this problem through a detailed comparison of the droplet size distributions obtained from the stochastic description for a finite system with the master equation (Eq. 2), and the deterministic approach for an infinite system by using the $\mathrm{KCE}$ (Eq. 1), using the numerical algorithm reported in $\mathrm{Al}$ fonso (2015). By the time the gel forms, certain differences are to be expected between the two approaches at the large end of the droplet size distribution.

This analysis of the sol-gel transition problem in the cloud physics context could provide an alternative explanation of the differences between modeled and observed droplet spectra in clouds. Several mechanisms have been proposed in the past (entrainment, presence of giant nuclei, supersaturation fluctuations, effects of air turbulence in concentration fluctuations and collision efficiencies, effects of film forming compounds on droplet growth), and a large amount of literature exists regarding the variety of mechanisms that may explain this disparity, but a conclusive answer is still absent. This study does not attempt to dispute any of the mechanisms already proposed, but to explore another mechanism that has not yet been widely considered in the mainstream literature.

The paper is organized as follows. Sect. 2 presents an overview of the numerical algorithm (following Alfonso, 2015). Numerical results (for the product and hydrodynamic kernels, respectively) with a detailed analysis of the method for calculating the sol-gel transition time and a comparison with averages calculated with the KCE are presented in Sects. 3 and 4. Finally, Sect. 5 presents a discussion of the limits of applicability of the KCE and an example of correlations in the critical region and conclusions.

\section{Overview of previous results: numerical solution of the master equation}

The objective of this section is to present a description of the algorithm. A more detailed explanation of the method can be found in Alfonso (2015), and only a brief summary is presented here. The main idea of the algorithm consists of the numerical calculation of all states for a given initial configuration with probability $P\left(n_{01}, n_{02}, \ldots, n_{0 N} ; 0\right)=1$, and the subsequent calculation of the temporal evolution of each 


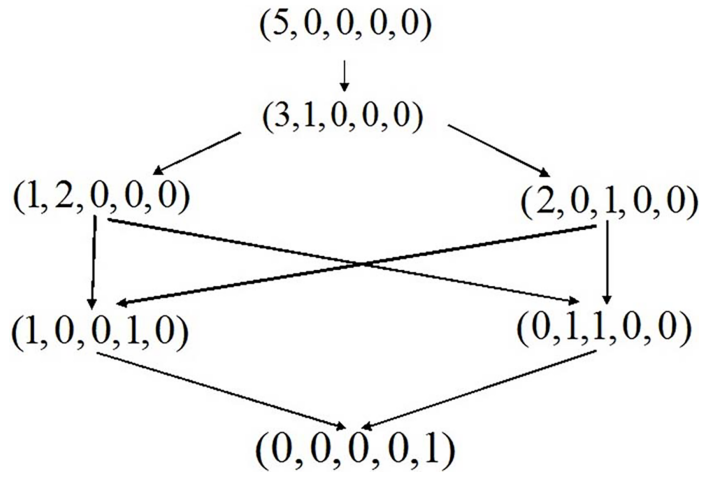

Figure 1. State space obtained from the initial condition $P(5,0,0,0,0 ; 0)=1$ with the constraint $\sum_{i=1}^{6} i n_{i}=5$.

state. The time evolution can be performed by considering that the only allowed transitions are of the form $\bar{n}_{1}^{(+)} \rightarrow \bar{n}_{1}$ if $i \neq j$ and $\bar{n}_{2}^{(+)} \rightarrow \bar{n}_{2}$ if $i=j$, where $\bar{n}_{1}^{(+)}, \bar{n}_{1}$ and $\bar{n}_{2}^{(+)}, \bar{n}_{2}$ are the state vectors:

$\bar{n}_{1}^{(+)}=\left(n_{1}, \ldots, n_{i}+1, \ldots, n_{j}+1, \ldots, n_{i+j}-1, \ldots, n_{N}\right)$

$\bar{n}_{1}=\left(n_{1}, \ldots, n_{i}, \ldots, n_{j}, \ldots, n_{i+j}, \ldots, n_{N}\right)$

$\bar{n}_{2}^{(+)}=\left(n_{1}, \ldots, n_{i}+2, \ldots, n_{2 i}-1, \ldots, n_{N}\right)$

$\bar{n}_{2}=\left(n_{1}, \ldots, n_{i}, \ldots, n_{2 i}, \ldots, n_{N}\right)$.

For a system consisting of $N$ monomers at $t=0$, the number of possible configurations increases exponentially and can be approximated from the equation (Hall, 1967):

$R(N) \approx \frac{1}{4 N \sqrt{3}} \exp \left(\pi(2 N / 3)^{1 / 2}\right)$

For example, $R(50)=217590$ and $R(100)=190569232$.

The procedure is illustrated for a system with five monomers in the initial state, only for the purpose of demonstrating the method. As the system in this case has only six possible states, it is much easier to explain the details of the calculations. The six possible configurations generated from the initial state $(5,0,0,0,0)$ are displayed in Fig. 1.

In a second step, the probabilities of all generated configurations are updated according to the first order finite differ- ence scheme (Alfonso, 2015):

$$
\begin{aligned}
P\left(\bar{n} ; t_{0}\right. & +\Delta t)=P\left(\bar{n} ; t_{0}\right) \\
& +\Delta t \sum_{i=1}^{N} \sum_{j=i+1}^{N} K(i, j)\left(n_{i}+1\right)\left(n_{j}+1\right) \\
& \times P\left(\ldots, n_{i}+1, \ldots, n_{j}\right. \\
& \left.+1, \ldots, n_{i+j}-1, \ldots ; t_{0}\right) \\
& +\Delta t \sum_{i=1}^{N} \frac{1}{2} K(i, i)\left(n_{i}+2\right)\left(n_{i}+1\right) \\
& \times P\left(\ldots, n_{i}+2, \ldots, n_{2 i}-1, \ldots ; t_{0}\right) \\
& -\Delta t \sum_{i, j=1}^{N} K(i, j) n_{i} n_{j} P\left(\bar{n} ; t_{0}\right) \\
& -\Delta t \sum_{i=1}^{N} \frac{1}{2} K(i, i) n_{i}\left(n_{i}-1\right) P\left(\bar{n} ; t_{0}\right) .
\end{aligned}
$$

From Eq. (5) it should be clear that the state probabilities $P\left(\bar{n} ; t_{0}+\Delta t\right)$ at $t=t_{0}+\Delta \mathrm{t}$ will increase if the states from which transitions are allowed have a nonzero probability at $t=t_{0}$ (second and third terms in the right-hand side of Eq. 5), and will decrease due to collisions of particles from the same state at $t=t_{0}$ (fourth term and fifth terms in the right-hand side of Eq. 5) if $P\left(\bar{n} ; t_{0}\right)$ is positive.

The finite difference equation for $P(1,0,0,1,0)$ is presented to illustrate the method. From the generation scheme displayed in Fig. 1, note that the only allowed transitions to $(1,0,0,1,0)$ are from the states $(1,2,0,0,0)$ and $(2,0,1,0,0)$. Consequently, at $t=t_{0}+\Delta t$, $P\left(1,0,0,1,0 ; t_{0}+\Delta t\right)$ will increase if $P\left(1,2,0,0,0 ; t_{0}\right)$ and $P\left(2,0,1,0,0 ; t_{0}\right)$ are positive at $t=t_{0}$. On the other hand, $P\left(1,0,0,1,0 ; t_{0}+\Delta t\right)$ will decrease due to collisions from particles within the same state at $t=t_{0}$ if $P\left(1,0,0,1,0 ; t_{0}\right)$ is positive. Then, $P\left(1,0,0,1,0 ; t_{0}+\Delta t\right)$ is calculated from the following equation:

$$
\begin{aligned}
& P\left(1,0,0,1,0 ; t_{0}+\Delta t\right)=P\left(1,0,0,1,0 ; t_{0}\right) \\
& \quad+\Delta t(1 / 2) K(2,2)\left(n_{2}+2\right)\left(n_{2}+1\right) \\
& \quad \times P\left(1,2,0,0,0 ; t_{0}\right) \\
& \quad+\Delta t K(1,3)\left(n_{1}+1\right)\left(n_{3}+1\right) P\left(2,0,1,0,0 ; t_{0}\right) \\
& \quad-\Delta t K(1,4)\left(n_{1}\right)\left(n_{4}\right) P\left(1,0,0,1,0 ; t_{0}\right) .
\end{aligned}
$$

The time evolution of the probability of each state was calculated for the product kernel $K(i, j)=C x_{i} x_{j}$, considering $C=5.49 \times 10^{10} \mathrm{~cm}^{3} \mathrm{~g}^{-2} \mathrm{~s}^{-1}$ following Long (1974) and for the initial condition $P(5,0,0,0,0 ; 0)=1$. Due to the small number of droplets in the initial configuration (only 5), the simulated volume was set equal to $10^{-2} \mathrm{~cm}^{3}$, with an initial droplet radius of $17 \mu \mathrm{m}$. The time step was $\Delta t=0.1 \mathrm{~s}$. For this case, the time evolution of four of the seven configurations is displayed in Fig. 2.

After the calculation for each state is completed, the expected values for each droplet mass can be found from the following relation (Alfonso, 2015):

$\left\langle n_{\mathrm{m}}\right\rangle=\sum_{n} n P(n, m ; t)$, 


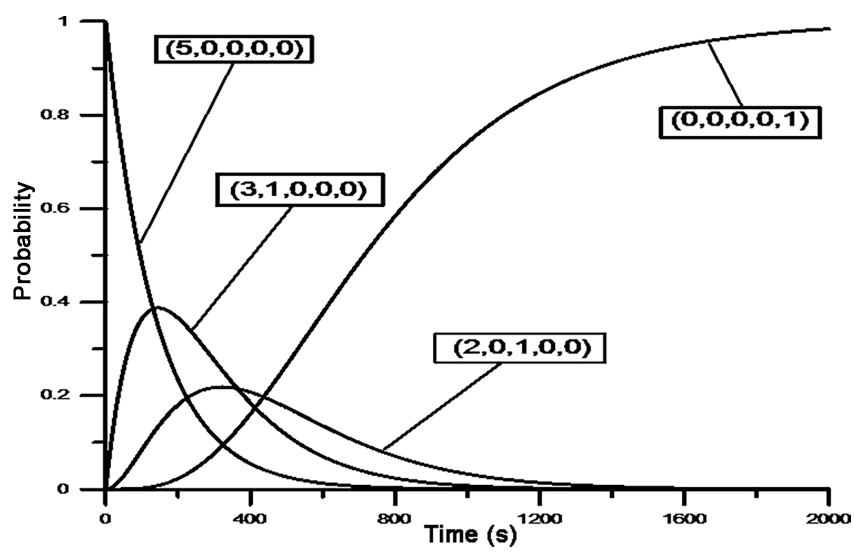

Figure 2. Time evolution of the probabilities of 4 of the 7 states for the initial condition $P(5,0,0,0,0 ; 0)=1$. Simulations were performed with the collection kernel $K(i, j)=C x_{i} x_{j}$ (with $C=$ $\left.5.49 \times 10^{10} \mathrm{~cm}^{3} \mathrm{~g}^{-2} \mathrm{~s}^{-1}\right)$.

where the discrete probability mass function is calculated from the state probabilities following the expression:

$$
\begin{aligned}
& P(n, m ; t)=\sum_{\text {All states with } n_{\mathrm{m}}=n} \\
& P\left(n_{1}, n_{2}, \ldots, n_{\mathrm{m}}=n, \ldots n_{N} ; t\right) .
\end{aligned}
$$

The expected values $\left\langle n_{\mathrm{m}}\right\rangle$ calculated from Eq. (7) are the magnitudes that must be compared with the averages $N(m, t)$ obtained from the KCE (Eq. 1).

\section{Results for the multiplicative kernel}

\subsection{Estimating the time of gel formation}

Lushnikov (2004) demonstrated that right after the sol-gel transition, the particle mass distribution splits into two parts: the thermodynamically populated one with behavior described by the kinetic collection equation, and a narrow peak with a mass very close to the gel mass. For the infinite system described by the KCE (Eq. 1) with kernel $K(i, j)=C x_{i} x_{j}$, the critical time is calculated when the second moment of the distribution diverges,

$$
M_{2}(\tau)=\frac{M_{2}\left(t_{0}\right)}{1-C M_{2}\left(t_{0}\right) \tau},
$$

leading to the critical time of the sol-gel transition:

$$
T_{\text {gel }}=\left[C M_{2}\left(t_{0}\right)\right]^{-1} \text {. }
$$

After $\tau=T_{\text {gel }}$ the second moment becomes undefined, and the total mass of the system starts to decrease.

For a finite system, the standard deviation $(\sigma)$ of the mass of the largest droplet is important for calculating the critical time of the gel formation (Botet, 2011). At the critical time of

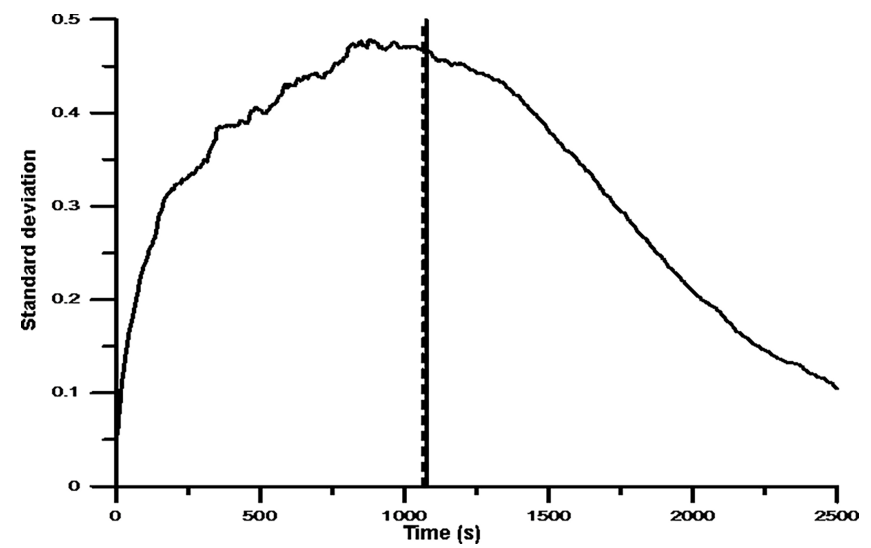

Figure 3. For the finite system, the relative standard deviation $\sigma\left(S_{\max }\right)$ of the largest droplet mass versus time. The initial number of droplets was set equal to $N=40$ droplets of $17 \mu \mathrm{m}$ in radius in a volume of $1 \mathrm{~cm}^{3}$. Simulations were performed with the product kernel $K(i, j)=C x_{i} x_{j}$ (with $\left.C=5.49 \times 10^{10} \mathrm{~cm}^{3} \mathrm{~g}^{-2} \mathrm{~s}^{-1}\right)$, and $N_{\mathrm{r}}=2000$ realizations of the stochastic algorithm were performed. The maximum value of $\sigma\left(S_{\max }\right)$ is found to be $1065 \mathrm{~s}$. (dashed vertical line) and is very close to the sol gel transition time (continuous vertical line) for the infinite system (1075 s.).

the infinite system, $\sigma$ must diverge, since it is proportional to the second moment of the distribution $M_{2}(\tau)$ which diverges at the gelation point. However, for a finite system (with no critical behavior), the relative standard deviation (standard deviation of mass divided by mean mass) of the mass of the largest droplet $\sigma\left(S_{\max }\right)$ is expected to reach maximum for a time close to $T_{\mathrm{gel}}=\left[C M_{2}\left(t_{0}\right)\right]^{-1}$.

This was explored in previous studies (Inaba, 1999; Alfonso et al., 2008, 2010, 2013), where $\sigma$ was calculated for a finite system from Monte Carlo simulations in order to estimate the sol-gel transition times for the corresponding deterministic model of an infinite system. We can perform an example calculation of $\sigma$ by using the species formulation of the SSA (Laurenzi and Diamond, 2002), in this case:

$\sigma\left(S_{\max }\right)=\sqrt{\frac{1}{N_{\mathrm{r}}} \sum_{i=1}^{N_{\mathrm{r}}}\left(S_{\max }^{i}-\left\langle S_{\max }\right\rangle\right)^{2}}$,

where $S_{\max }^{i}$ is the value of $S_{\max }=M_{\max } /\left\langle M_{\max }\right\rangle$ for each realization at a given time, and $N_{\mathrm{r}}$ is the number of iterations of the SSA. $M_{\max }$ is the size of the largest particle, and $\left\langle M_{\max }\right\rangle$ its ensemble mean over all the realizations. The time evolution of $\sigma\left(S_{\max }\right)$ is shown in Fig. 3 for a finite system with $N=40$ droplets of $17 \mu \mathrm{m}$ in radius (droplet mass $=2.058 \times 10^{-8} \mathrm{~g}$ ) in a volume of $1 \mathrm{~cm}^{3}$. For the product kernel with $C=5.49 \times 10^{10} \mathrm{~cm}^{3} \mathrm{~g}^{-2} \mathrm{~s}^{-1}$, the maximum occurs at $T=1065 \mathrm{~s}$, which is close to the sol-gel transition time $T_{\text {gel }}=1075 \mathrm{~s}$. 


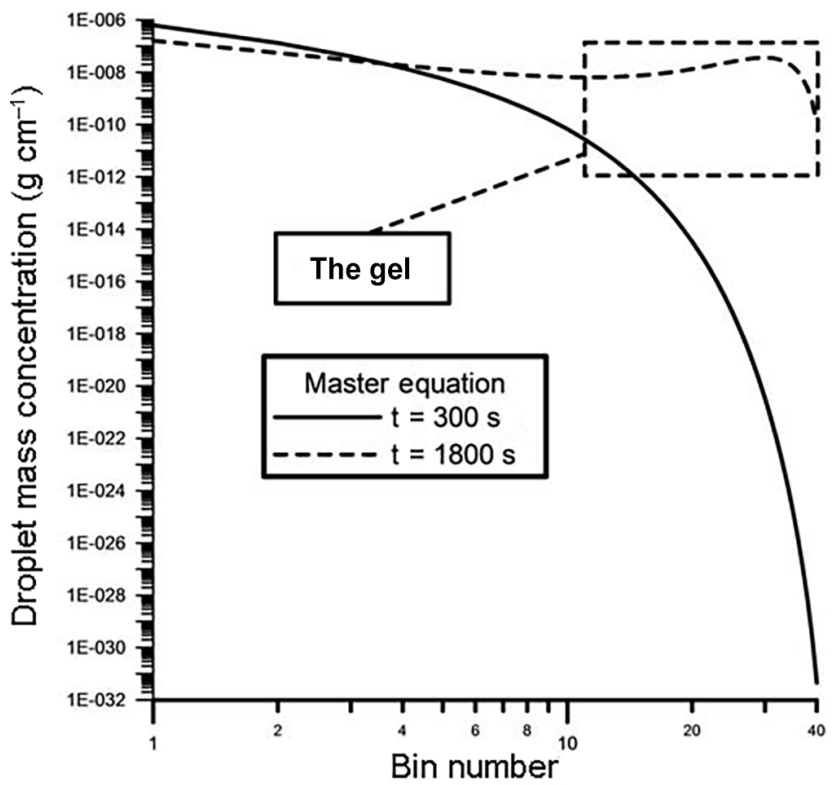

(a)

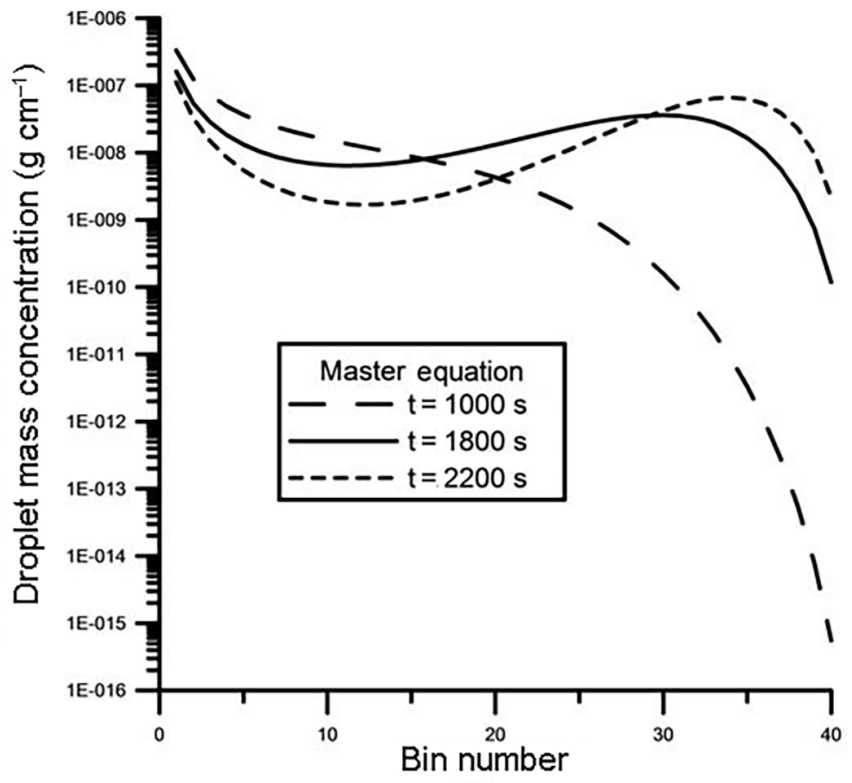

(b)

Figure 4. The droplet mass spectrum at different times $(t=300,1000,1800$ and $2200 \mathrm{~s}$.). The gel is clearly observed at $t=1800,2200 \mathrm{~s}$. Simulations were performed with the collection kernel $K(i, j)=C x_{i} x_{j}$ (with $C=5.49 \times 10^{10} \mathrm{~cm}^{3} \mathrm{~g}^{-2} \mathrm{~s}^{-1}$ ). The initial number of droplets was set equal to $N=40$ droplets of $17 \mu \mathrm{m}$ in radius in a volume of $1 \mathrm{~cm}^{3}$.

\subsection{Calculation of the post-gel droplet size distribution from the master equation and comparison with the deterministic (kinetic) approach}

The evolution of a system with an initial monodisperse droplet size distribution of $N_{0}=40$ droplets of $17 \mu \mathrm{m}$ in radius at $t_{0}$, in a volume of $1 \mathrm{~cm}^{3}$, and a corresponding liquid water content (LWC) of $0.823 \mathrm{~g} \mathrm{~m}^{-3}$, was calculated using the master equation (Eq. 2). The initial condition for this case is $P(40,0, \ldots, 0 ; 0)=1$ and the time step was set equal to $\Delta t=0.1 \mathrm{~s}$. The complete phase space of a population with $N=40$ monomers contains 37338 states, and the master equation must be solved for the 37338 states. Then, for each state there is a finite difference Eq. (5) or an equation similar to Eq. (6), but with 40-D state vectors.

A discrete 40 bin grid was defined for our model. The mass for bin 1 is taken to be the mass of a $17 \mu \mathrm{m}$ in radius droplet, and the mass of bin $n$ is $n$ times the mass of bin 1 . Then, if all 40 droplets in the initial distribution were to coalesce into a single droplet, the final droplet radius would be $58.14 \mu \mathrm{m}$ in diameter and would belong to the mass bin 40 .

The results for the droplet mass distribution are displayed in Fig. 4 at $t=300,1000,1800$ and 2200 s. Note that the gel is clearly seen in the distributions at 1800 and $2200 \mathrm{~s}$ but not at $1000 \mathrm{~s}$.

To proceed further, the previous results are compared to the analytical size distributions from the KCE (Eq. 1) calculated for the product kernel with monodisperse initial conditions before $(t=300 \mathrm{~s})$ and after $(t=1200 \mathrm{~s})$ gel formation
(Laurenzi and Diamond, 2002):

$N(i, t)=N_{0} \frac{(i T)^{i-1}}{i \Gamma(i+1)} \exp (-i T)$ where $T=C N_{0} v_{0}^{2} t$.

In Eq. (12), $N_{0}=40$ is the initial concentration and $v_{0}$ is the initial volume of droplets. The index $i$ represents the bin size and $C=5.49 \times 10^{10} \mathrm{~cm}^{3} \mathrm{~g}^{-2} \mathrm{~s}^{-1}$.

The comparison of the droplet mass concentration $\left(g_{\mathrm{KCE}}(m)=N(m, t) m\right)$ calculated from Eq. (12), with expected values $\left(g_{\text {STOC }}(m)=\left\langle n_{\mathrm{m}}\right\rangle m\right)$ is calculated from the master equation (Eq. 2) with the same initial conditions are displayed in Fig. 5 at $t=300,1200 \mathrm{~s}$. Note that the KCE fails to capture the gel formation after the critical time, and the droplet mass concentration calculated using the kinetic approach is much lower at the large end of the distribution. This is due to the fact that the total mass calculated according to Eq. (12) decreases after the sol-gel transition time. This decrease can be clearly seen in the time evolution of the LWC from the kinetic approach using the relation:

$M_{1}(t)=\sum_{i=1}^{\infty} m(i) N(i, t)$

where $m(i)$ is the mass for bin size $i$. After $t \sim 1000 \mathrm{~s}$ (Fig. 6) the total mass of the system calculated according to the KCE starts to decrease, while the total mass calculated from the stochastic approach is conserved at all times. 


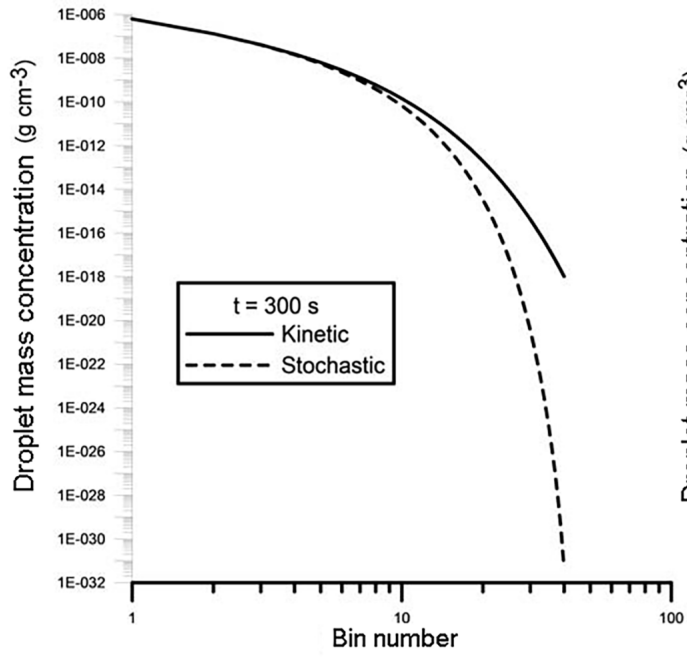

(a)

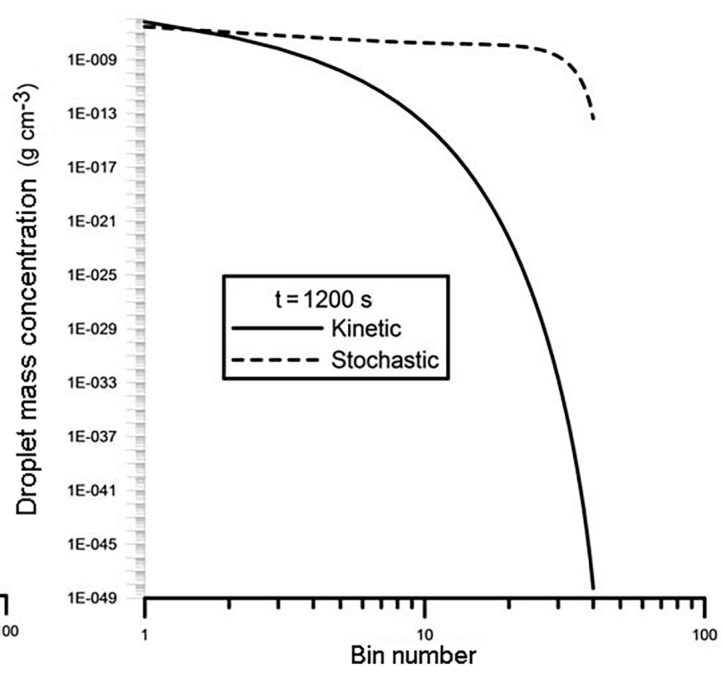

(b)

Figure 5. Size distributions obtained from the stochastic master equation (dashed lines) and the KCE (solid lines) at (a) $t=300 \mathrm{~s}$ and (b) $t=1200 \mathrm{~s}$. Simulations were performed with the collection kernel $K(i, j)=C x_{i} x_{j}$ (with $C=5.49 \times 10^{10} \mathrm{~cm}^{3} \mathrm{~g}^{-2} \mathrm{~s}^{-1}$ ). The initial number of droplets was set equal to $N=40$ droplets of $17 \mu \mathrm{m}$ in radius in a volume of $1 \mathrm{~cm}^{3}$. For the large end, the stochastic approach shows larger values of the drop mass concentration after the sol-gel transition.

\subsection{Calculation of the gel mass}

Within the master equation approach, the expected value of the mass of the largest droplet $M_{1}$ is approximately given by (Tanaka and Nakazawa, 1994):

$M_{1}=\sum_{i=i_{1}}^{N} m(i)\left\langle n_{i}\right\rangle$,

where $\left\langle n_{i}\right\rangle$ is the expected number for each droplet size value calculated from Eq. (7), $m(i)$ is the mass for bin size $i$, and the bin number $i_{1}$ is defined from the following relation:

$\sum_{i=i_{1}}^{N}\left\langle n_{i}\right\rangle=1$.

The mass of the gel, $M_{1}$, is evaluated for $t=1200,1800$ and 2200 s from Eqs. (14) and (15).

Within the Monte Carlo stochastic approach (SSA), the expected mass of the gel is the ensemble mean $\left(M_{1_{M C}}\right)$ calculated over all realizations $\left(N_{\mathrm{r}}\right)$ of the mass of the largest droplet (Alfonso et al., 2008):

$M_{1_{M C}}=\frac{1}{N_{\mathrm{r}}} \sum_{i=1}^{N_{\mathrm{r}}} M_{1}^{i}$,

where $N_{\mathrm{r}}=1000$ for this simulation and $M_{1}^{i}$ is the largest droplet for each realization. The results obtained from both the master equation and the SSA are displayed in Table 1, showing a very good correspondence between the two approaches.
Table 1. Expected gel mass calculated from the SSA, the master equation and the kinetic approach. Simulations were performed for the product kernel.

\begin{tabular}{lrrr}
\hline $\begin{array}{l}\text { Time } \\
(\mathrm{s})\end{array}$ & $\begin{array}{r}\text { Gel mass } \\
\text { (SSA) }\end{array}$ & $\begin{array}{r}\text { Gel mass } \\
\text { (master } \\
\text { equation) }\end{array}$ & $\begin{array}{r}\text { Gel mass } \\
\text { (KCE) }\end{array}$ \\
\hline 1200 & $2.84 \times 10^{-7} \mathrm{~g}$ & $2.76 \times 10^{-7} \mathrm{~g}$ & $1.75 \times 10^{-7} \mathrm{~g}$ \\
1800 & $5.14 \times 10^{-7} \mathrm{~g}$ & $5.34 \times 10^{-7} \mathrm{~g}$ & $5.59 \times 10^{-7} \mathrm{~g}$ \\
2200 & $6.63 \times 10^{-7} \mathrm{~g}$ & $6.35 \times 10^{-7} \mathrm{~g}$ & $6.66 \times 10^{-7} \mathrm{~g}$ \\
\hline
\end{tabular}

After the sol-gel transition, the mass of the gel can also be estimated by using the infinite system approach from the following relation (Wetherill, 1990):

$M_{\mathrm{gel}}=M_{0}-M_{\mathrm{KCE}}$,

where $M_{0}$ is the initial mass of the system, and $M_{\mathrm{KCE}}$ is the mass calculated from Eq. (13). The results shown in the third column of Table 1 indicate good agreement away from the sol-gel transition time.

\section{Results for the hydrodynamic collection kernel}

Collisions between droplets under pure gravity conditions are simulated with the hydrodynamic kernel, which has the following expression:

$K_{g}\left(x_{i}, x_{j}\right)=\pi\left(r_{i}+r_{j}\right)^{2}\left|V\left(x_{i}\right)-V\left(x_{j}\right)\right| E\left(r_{i}, r_{j}\right)$. 
Table 2. Expected gel mass calculated from the SSA and the master equation. Simulations were performed for the hydrodynamic kernel.

\begin{tabular}{lrrr}
\hline $\begin{array}{l}\text { Time } \\
(\mathrm{s})\end{array}$ & $\begin{array}{r}\text { Gel mass } \\
(\mathrm{SSA})\end{array}$ & $\begin{array}{r}\text { Gel mass } \\
\text { (master } \\
\text { equation) }\end{array}$ & $\begin{array}{r}\text { Gel mass } \\
(\mathrm{KCE})\end{array}$ \\
\hline $1200 \mathrm{~s}$ & $1.71 \times 10^{-7} \mathrm{~g}$ & $1.79 \times 10^{-7} \mathrm{~g}$ & $3.21 \times 10^{-8} \mathrm{~g}$ \\
$1800 \mathrm{~s}$ & $3.34 \times 10^{-7} \mathrm{~g}$ & $3.37 \times 10^{-7} \mathrm{~g}$ & $3.63 \times 10^{-7} \mathrm{~g}$ \\
$2200 \mathrm{~s}$ & $4.35 \times 10^{-7} \mathrm{~g}$ & $4.68 \times 10^{-7} \mathrm{~g}$ & $5.14 \times 10^{-7} \mathrm{~g}$ \\
\hline
\end{tabular}

The hydrodynamic kernel takes into account the fact that droplets with different masses ( $x_{i}$ and $x_{j}$ and corresponding radii, $r_{i}$ and $r_{j}$ ) have different terminal velocities, which are functions of their masses. In Eq. (18), $E\left(r_{i}, r_{j}\right)$ are the collection efficiencies calculated according to Hall (1980).

\subsection{Estimating the time of gel formation and the gel mass}

For an infinite system modeled by the KCE (Eq. 1) with the hydrodynamic kernel, the second moment of the mass distribution $\left(M_{2}\right)$ diverges when the raindrop embryo (gel) forms. As there is no a simple analytical expression to calculate the critical time (see Eq. 9 for the product kernel) in this case, Monte Carlo simulations for the finite system could provide insightful information.

The sol-gel transition time can be estimated approximately by calculating the time at which the time series of $\sigma\left(S_{\max }\right)$ in the SSA exhibits a maximum (Alfonso et al., 2010). As in the case of multiplicative kernel, the time evolution of $\sigma\left(S_{\max }\right)$ is calculated for a cloud volume of $1 \mathrm{~cm}^{3}$ with an initial bidisperse distribution (20 droplets of $17 \mu \mathrm{m}$ in radius and 10 droplets of $21.4 \mu \mathrm{m}$ ), and the time evolution of $\sigma\left(S_{\max }\right)$ is calculated from 1000 realizations $\left(N_{\mathrm{r}}=1000\right)$ of the SSA. Figure 7 shows that there is a maximum at $t=1310 \mathrm{~s}$, which is considered a good estimate for the solgel transition time for the infinite system. Thus, the distributions obtained from the stochastic (master equation) and the deterministic (kinetic collection equation) approaches must be compared before and after $1310 \mathrm{~s}$. After the critical time, the gel mass was calculated using Eqs. (14) and (15). The results are displayed in Table 2, again showing a good agreement between the calculations performed with the SSA and the master equation.

Although for the hydrodynamic kernel the critical time was longer than $20 \mathrm{~min}$, we must emphasize that, in general, for concentrations larger than $30-40 \mathrm{~cm}^{-3}$, smaller critical times must be obtained. For example, for kernels proportional to the product of the masses, Malyshkin and Goldman (2001) demonstrated that for monodisperse initial distributions the critical time decreases as a power of the logarithm of the initial number of particles $\tau_{\text {critical }} \sim 1 / \log \left(\mathrm{N}_{0}\right)$. For kernels relevant to cloud physics, we have a similar situation (a decrease in the time of occurrence of the phase tran-

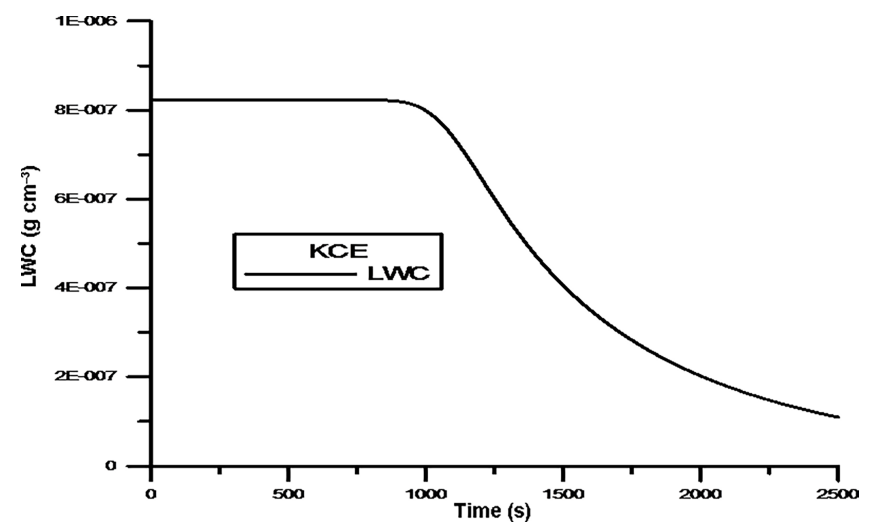

Figure 6. Time evolution of the total liquid water content calculated from the analytical solution of the kinetic collection equation for the product kernel.

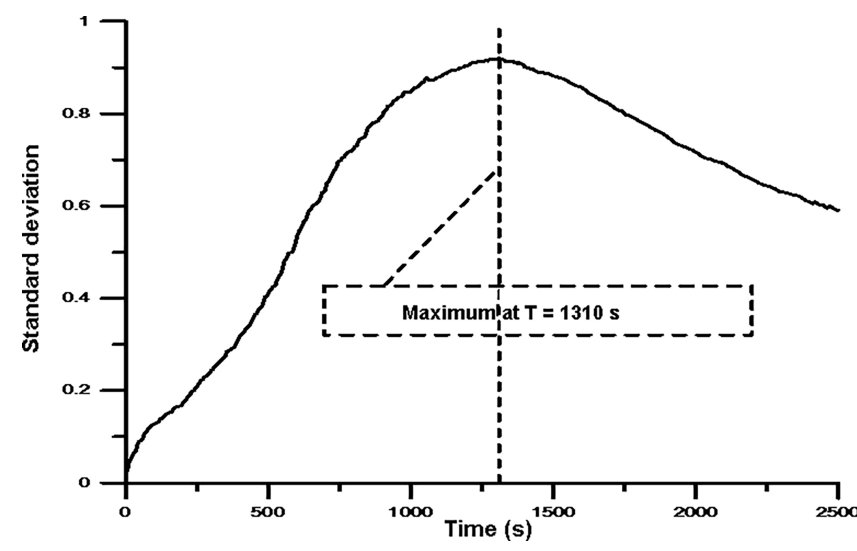

Figure 7. Time evolution of the relative standard deviation $\sigma\left(S_{\max }\right)$ of the mass of the largest droplet, for a finite system modeled with the hydrodynamic collection kernel. The initial distribution was bidisperse with 20 droplets of $17 \mu \mathrm{m}$ in radius and 10 droplets of $21.4 \mu \mathrm{m}$ in a volume of $1 \mathrm{~cm}^{3}$. The maximum of $\sigma\left(S_{\max }\right)$ was found at $1310 \mathrm{~s}$.

sition as the number of particles in the initial distribution increases). A more detailed discussion of this problem for realistic collection kernels can be found in Alfonso et al. (2010, 2013).

\subsection{Calculation of the post-gel droplet size distribution from the master equation and comparison with the deterministic (kinetic) approach}

The evolution of a system with the initial bidisperse droplet size distribution described in the previous section is calculated here using the master equation (Eq. 2) with the initial condition $P(20,10, \ldots, 0 ; 0)=1$ and a time step $\Delta t=0.1 \mathrm{~s}$. The results for the expected droplet mass distribution as a function of radius are displayed in Fig. 8 at four different times $(t=500,1500,1800$ and $2500 \mathrm{~s})$. 


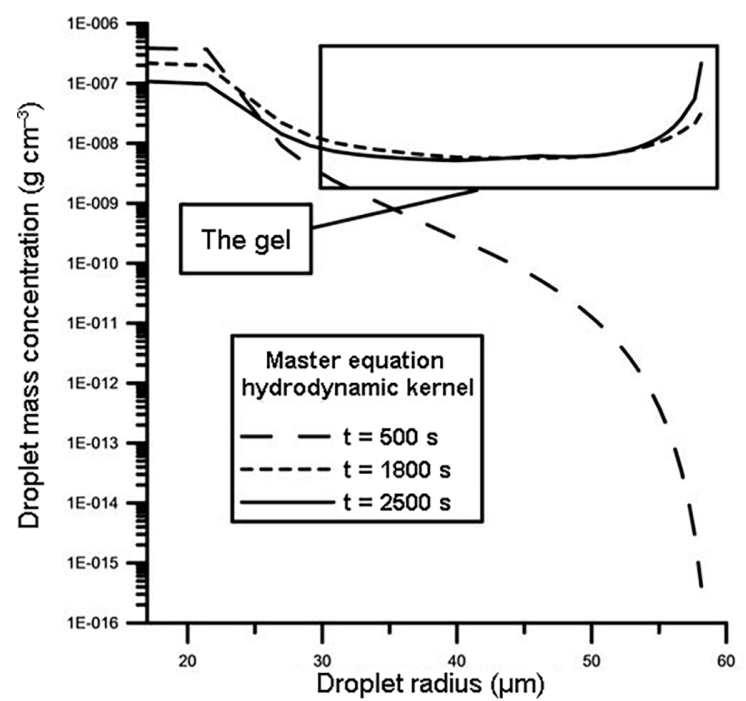

(a)

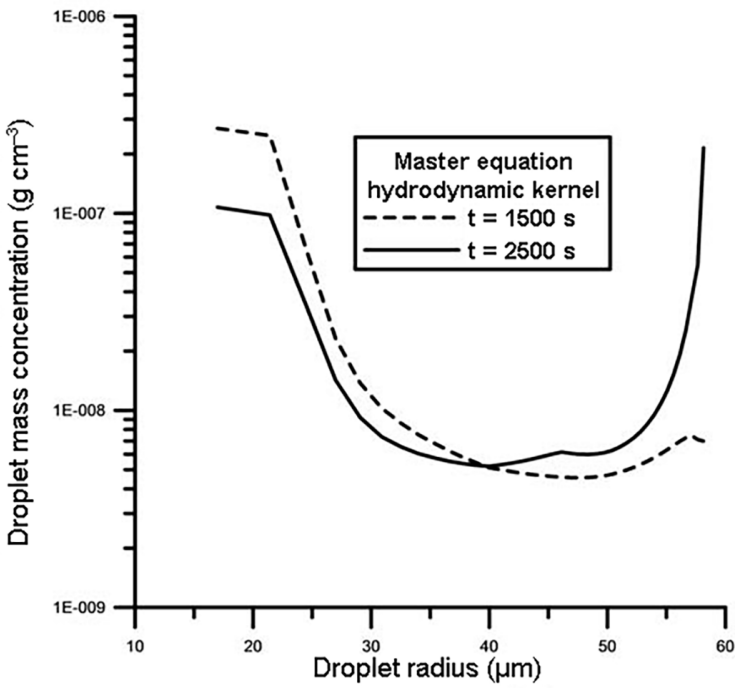

(b)

Figure 8. The droplet mass spectrum at different times $(t=500,1500,1800$ and $2500 \mathrm{~s})$ for a finite system modeled with the hydrodynamic collection kernel. The initial distribution is bidisperse with 20 droplets of $17 \mu \mathrm{m}$ in radius and 10 droplets of $21.4 \mu \mathrm{m}$ in a volume of $1 \mathrm{~cm}^{3}$.

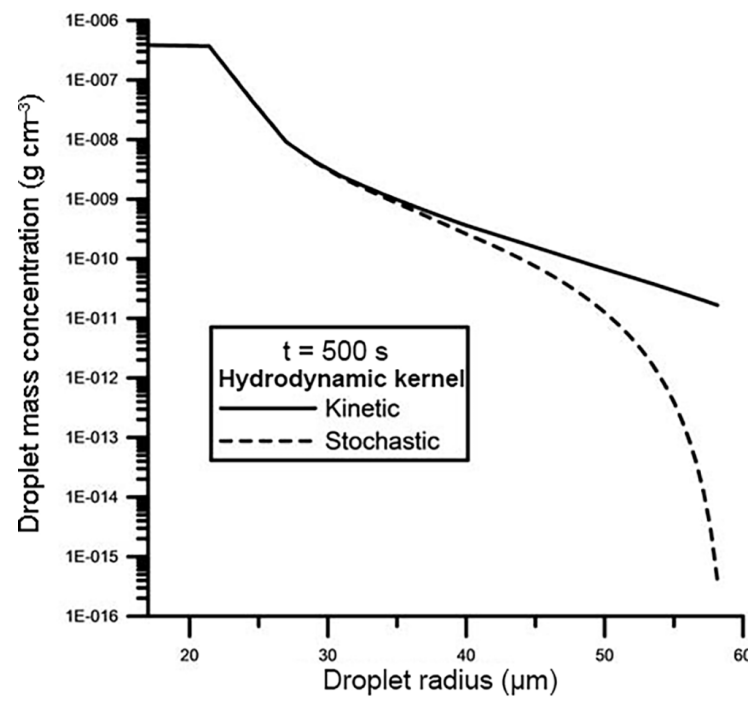

(a)

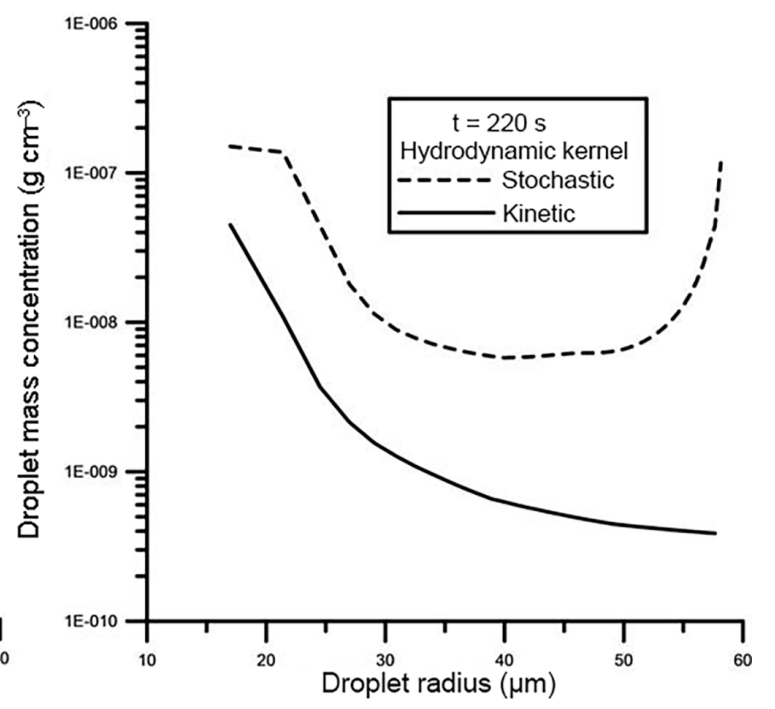

(b)

Figure 9. Comparison of the size distributions obtained from the stochastic master equation (dashed lines) with that to the KCE (solid lines) at $t=500 \mathrm{~s}$ in (a) and $t=2200 \mathrm{~s}$ in (b) for the hydrodynamic kernel. The initial distribution was bidisperse with 20 droplets of $17 \mu \mathrm{m}$ in radius and 10 droplets of $21.4 \mu \mathrm{m}$ in a volume of $1 \mathrm{~cm}^{3}$.

Before the sol-gel transition, the mass spectrum exponentially decreases with increasing drop radius for both the KCE and the master equation. After the sol-gel transition, there are two types of behavior in the droplet mass distribution of the master equation: (i) an exponential decay that resembles the KCE description, and (ii) a peak in the gel fraction of the distribution, in which the mass is calculated according to Eqs. (14) and (15). As can be observed in Fig. 9, there are substantial differences between the kinetic and the stochastic approaches, especially in the large end of the distribution after the critical time, with much higher values of the droplet mass concentration for the stochastic case.

\section{Discussion and conclusions}

In their pioneering studies using the stochastic framework, Marcus (1968) and Bayewitz et al. (1974) solved the stochas- 


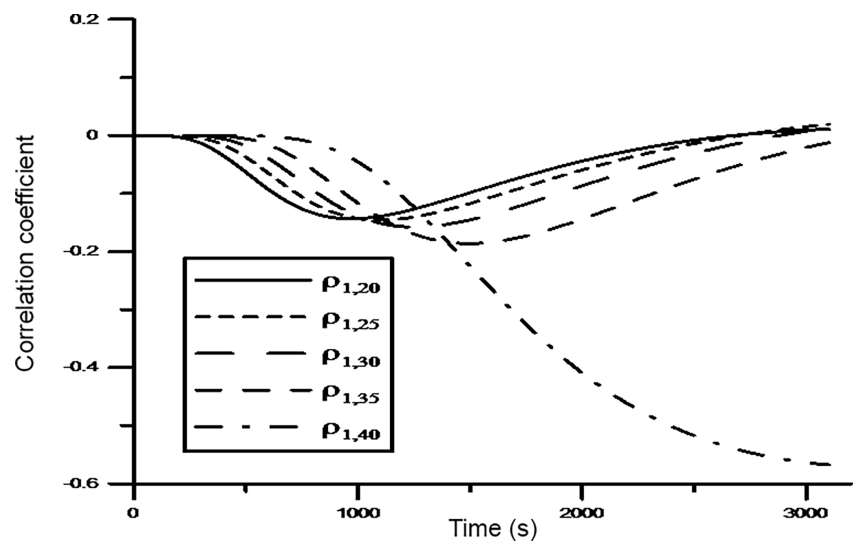

Figure 10. Time evolution of the correlation coefficients for different bin pairs $\rho_{1,20}, \rho_{1,25}, \rho_{1,30}, \rho_{1,35}$ and $\rho_{1,40}$ for a $1 \mathrm{~cm}^{3}$ system modeled with the hydrodynamic kernel, and containing initially 20 droplets of $17 \mu \mathrm{m}$ and 10 droplets of $21.4 \mu \mathrm{m}$.

tic master equation (Eq. 2) for a constant collection kernel and a monodisperse initial droplet distribution. The latter study revealed significant deviations from the KCE when there is a small number of droplets in the initial distribution $\left(\left\langle N_{\text {Total }}\right\rangle<50\right)$. In our work, we extended the results obtained by these authors by calculating the expected droplet size distributions for small systems within the stochastic framework, but by using collection kernels that are mass dependent and relevant to cloud physics (e.g. multiplicative and hydrodynamic). Our results confirm the findings of Bayewitz et al. (1974) that in systems of small populations the results of the kinetic deterministic equations approach may differ substantially from the stochastic means at the large end of the droplet size distribution.

The application of the KCE to coagulating systems also requires that the particles are well mixed (Bayewitz et al., 1974; Sampson and Ramkrishna, 1985), implying that every pair of droplets is always available for coagulation (Sampson and Ramkrishna, 1985).

Another important assumption is that the droplet population is sufficiently large for the existence of a droplet with particular properties to not be conditionally dependent on the existence or nonexistence of any other droplet. In other words, no correlations are assumed in the system, so that $\left\langle n_{i} n_{j}\right\rangle=\left\langle n_{i}\right\rangle\left\langle n_{j}\right\rangle$.

The assumption that the system is sufficiently large is linked to the fact that the KCE is a deterministic equation that simulates only the mean values and gives an incomplete description of the coagulating system if fluctuations about the mean are very large (Ramkrishna and Borwanker, 1973). Since fluctuations are proportional to $\sim 1 / \sqrt{\left\langle N_{\text {Total }}\right\rangle}$, a large number of droplets is needed for the fluctuations to be small, and in fact, the larger the number of particles in a system, the smaller the fluctuations. This fact underscores the finite system description adopted in this work, as the collision- coalescence process is limited to pairs of droplets in close proximity to each other. The KCE is not expected to be accurate when the number of droplets or the volume of the system are small.

Additionally, the KCE can fail even if the number of droplets is large when a raindrop embryo forms. At that critical time, there is a transition from a continuous droplet distribution to a continuous distribution plus a raindrop embryo (or runaway droplet). This sol-gel transition is well known in other fields (e.g. astronomy), but has not been sufficiently explored in the context of cloud physics, where the gel would correspond to the raindrop embryo. This approach is developed in this paper through a detailed comparison of expected values calculated from the stochastic framework with averages obtained from the KCE for realistic collection kernels, before and after the sol-gel transition time.

The marked differences between these two approaches at the sol-gel transition can be related to the increase of correlations at the critical point, and that can happen even for a large number of particles in the initial distribution (Malyshkin and Goldman, 2001). When the sol-gel transition occurs, the occupation numbers $n_{i}$ of all low-mass bins are strongly anticorrelated with bins from the gel fraction (calculated from Eq. 15). On the other hand, in the vicinity of critical time, the fluctuations for the finite system are larger, since the standard deviation of the mass of the largest droplet, $\sigma\left(S_{\max }\right)$, has a maximum. As a consequence, the differences between the deterministic and stochastic descriptions become larger and divergent. To further analyze this problem, the time evolution of the correlation coefficients

$\rho_{i, j}=\frac{\operatorname{cov}\left(n_{i}, n_{j}\right)}{\sqrt{\operatorname{Var}\left(n_{i}\right) \operatorname{Var}\left(n_{j}\right)}}=\frac{\sigma_{n_{i} n_{j}}}{\sigma_{n_{i}} \sigma_{n_{j}}}$

between the random variables $n_{1}$ and $n_{i}$ from bins within the gel fraction (see Eq. 15) were calculated. In the simulations, the gel fraction at $t=1310 \mathrm{~s}$ covers the interval bins from 29 to $58 \mu \mathrm{m}$, and narrows as time increases. The time evolution of the correlation coefficients for different bin pairs $\rho_{1,20}$, $\rho_{1,25}, \rho_{1,30}, \rho_{1,35}$ and $\rho_{1,40}$ are displayed in Fig. 10, showing, in all cases, an increase in the magnitude of the correlation coefficients in the vicinity of the sol-gel transition time. Also in Fig. 10, correlations are quite large in magnitude and negative most of the time for $\rho_{1,20}, \rho_{1,25}$ and $\rho_{1,30}$ as the mass of the gel increases through coalescence with droplets from low-mass bins. However, after $2500 \mathrm{~s}$, pairs 1-20, 1-25 and 1-30 are positively correlated as the gel fraction narrows and droplets from bins as large as 20,25 and 30 are also depleted by the gel. The correlation coefficients for all analyzed pairs have maxima between 1000 and $1500 \mathrm{~s}$, in the vicinity of the critical time. The random variable $n_{40}$ is always anticorrelated with $n_{1}$, with values much higher than the other pairs after $1500 \mathrm{~s}$, and increasing in magnitude until the end of the simulation, which reflects the fact that the gel actively grows by collecting smaller droplets. Thus, for realistic collection kernels, the mean values predicted by the KCE will be not 
accurate after the sol-gel transition. The stochastic approach captures the gel formation and evolution properly, with larger values of the expected droplet mass at the large end of the distribution.

In principle, this analysis could be performed by using the SSA, which is an alternative tool for the master equation formalism (Eq. 2). However, the number of realizations required to obtain smooth behavior at the large end in order to compare it with averages from the $\mathrm{KCE}$, would be extremely large.

It is necessary to emphasize that our method (although it can be computationally expensive) works for any type of kernels, whereas the analytical techniques developed by Lushnikov (2004) and Matsoukas (2015) work only for very special cases.

The failure of the KCE to capture the gel formation could provide an explanation of the inability of explicit microphysics cloud models to explain the droplet spectral broadening observed in small, warm clouds. Therefore, even for large simulation cells, the use of the KCE is justified only in the absence of the sol-gel transition.

For the small-volume approach described in this paper, a model that considers the interaction between small coalescence volumes through sedimentation or other physical mechanisms for realistic collection kernels is needed. For a constant collection kernel, this theory was outlined by Merkulovich and Stepanov $(1990,1991)$ based on a scheme proposed by Nicolis and Prigogine (1977) for chemical reactions. Within this theory, the whole system is subdivided into spatially homogeneous subvolumes (coalescence cells) that interact through the diffusion process, and the coalescence events are permitted only between droplets from the same subvolume. As a result, we obtain a set of master equations for each subvolume. Although very complex, it could be a starting point for considering the interactions between small coalescence volumes through different physical mechanisms.

Data availability. No data sets were used in this article.

Competing interests. The authors declare that they have no conflict of interest.

Acknowledgements. This study was funded by the grant "Estancias Sabáticas Nacionales" from CONACYT, in Mexico and it was completed during an academic visit at Centro de Ciencias de la Atmósfera, UNAM. L. Alfonso also thanks the Associate Program of the Abdus Salam International Center of Theoretical Physics (ICTP), in Trieste, for all the support provided for the completion of this paper during the summers of 2015 and 2016.

Edited by: G. Feingold

Reviewed by: two anonymous referees

\section{References}

Alfonso, L.: An algorithm for the numerical nsolution of the multivariate master equation for stochastic coalescence, Atmos. Chem. Phys., 15, 12315-12326, https://doi.org/10.5194/acp-1512315-2015, 2015.

Alfonso, L., Raga, G. B., and Baumgardner, D.: The validity of the kinetic collection equation revisited, Atmos. Chem. Phys., 8, 969-982, https://doi.org/10.5194/acp-8-969-2008, 2008.

Alfonso, L., Raga, G. B., and Baumgardner, D.: The validity of the kinetic collection equation revisited - Part 2: Simulations for the hydrodynamic kernel, Atmos. Chem. Phys., 10, 7189-7195, https://doi.org/10.5194/acp-10-7189-2010, 2010.

Alfonso, L., Raga, G. B., and Baumgardner, D.: The validity of the kinetic collection equation revisited - Part 3: Sol-gel transition under turbulent conditions, Atmos. Chem. Phys., 13, 521-529, https://doi.org/10.5194/acp-13-521-2013, 2013.

Bayewitz, M. H., Yerushalmi, J., Katz, S., and Shinnar, R.: The extent of correlations in a stochastic coalescence process, J. Atmos. Sci., 31, 1604-1614, 1974.

Botet, R.: Where are correlations hidden in the distribution of the largest fragment?, PoS, 7, WPCF2011, 2011.

Gillespie, D. T.: An Exact Method for Numerically Simulating the Stochastic Coalescence Process in a Cloud, J. Atmos. Sci. 32, 1977-1989, 1975.

Hall, M. J.: Combinatorial Theory. Blaisdell Pub. Co., 1967.

Hall, W. D.: A detailed microphysical model within a twodimensional dynamic framework: Model description and preliminary results, J. Atmos. Sci., 37, 2486-2507, 1980.

Inaba, S., Tanaka, H., Ohtsuki, K., and Nakazawa, K.: Highaccuracy statistical simulation of planetary accretion: I. Test of the accuracy by comparison with the solution to the stochastic coagulation equation, Earth Planet Space, 51, 205-217, 1999.

Laurenzi, I. J., Bartels, J. D., and Diamond, S. L.: A general algorithm for exact simulation of multicomponent aggregation processes, J. Comput. Phys., 177, 418-449, 2002.

Lushnikov, A. A.: Coagulation in finite systems, J. Colloid Interf. Sci., 65, 276-285, 1978.

Lushnikov, A. A.: From sol to gel exactly, Physi. Rev. Lett., 93, p. 198302, 2004.

Long, A. B.: Solutions to the droplet collection equation for polynomial kernels, J. Atmos. Sci., 31, 1040-1051, 1974.

Marcus, A. H.: Stochastic coalescence, Technometrics, 10, 133143, 1968.

Malyshkin, L. and Goodman, J.: The timescale of runaway stochastic coagulation, Icarus, 150, 314-322, 2001.

Matsoukas, Themis.: Statistical Thermodynamics of Irreversible Aggregation: The Sol-Gel Transition, Sci. Rep., 5, 8855, https://doi.org/10.1038/srep08855, 2015.

Nicolis, G. and Prigogine, L.: Self-Organization in NonEquilibrium systems, Willey, NY, 1977.

Merkulovich, V. M. and Stepanov, A. S.: Statistical description of coagulation in finite spatially inhomogeneous systems, Atmos. Res., 25, 431-444, 1990.

Merkulovich, V. M. and Stepanov, A. S.: Statistical description of coagulation in finite spatially inhomogenous systems (Part 2), Atmos. Res., 26, 311-327, 1991.

Pruppacher, H. R. and Klett, J. D.: Microphysics of clouds and precipitation, Kluwer Academic Publishers, 1997. 
Ramkrishna, D. and Borwanker, J. D.: A puristic analysis of population balance-I, Chem. Eng. Sci., 28, 1423-1435, 1973.

Sampson, K. J. and Ramkrishna, D.: Particle size correlations and the effects of limited mixing on agglomerating particulate systems, J. Colloid Interf. Sci., 104, 269-276, 1985.

Tanaka, H. and Nakazawa, K.: Stochastic coagulation equation and the validity of the statistical coagulation equation, J. Geomag. Geoelecr., 45, 361-381, 1993.
Tanaka, H. and Nakazawa, K.: Validity of the statistical coagulation equation and runaway growth of protoplanets, Icarus, 107, 404412, 1994.

Valioulis, I. A. and List, E. J.: A numerical evaluation of the stochastic completeness of the kinetic coagulation equation, J. Atmos. Sci., 41, 2516-2529, 1984.

Wetherill, G. W.: Comparison of analytical and physical modeling of planetesimal Accumulation, Icarus, 88, 336-354, 1990. 